



\section{Prácticas fisicodeportivas Actitudes y tendencias de escolares colombianos}





\section{Prácticas \\ fisicodeportivas}

Actitudes y tendencias
de escolares colombianos

Jesús Astolfo Romero García
Nelson Orlando Clavijo Gutiérrez 
Romero García, Jesús Astolfo

Prácticas fisicodeportivas. Actitudes y tendencias de escolares colombianos/ Jesús Astolfo Romero García y Nelson Orlando Clavijo Gutiérrez, Bogotá: Universidad Santo Tomás, 2019.

120 páginas; gráficos, ilustraciones y tablas

Incluye referencias bibliográficas (páginas 109-118)

ISBN: 978-958-782-262-5

E-ISBN: 978-958-782-263-2

1. Educación física-Enseñanza 2. Animación sociocultural 3. Deportistas-Aspectos psicológicos 4. Deportes Aspectos culturales 5. Gimnasia-Enseñanza 6. Deportes escolares -aspectos sociales. I. Universidad Santo Tomás (Colombia).

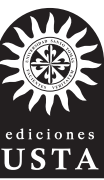

(C) Jesús Astolfo Romero García y Nelson Orlando Clavijo Gutiérrez, 2019

(C) Universidad Santo Tomás, 2019

\section{Ediciones USTA}

Bogotá, D. C., Colombia

Carrera 9 n. ${ }^{\circ} 51-11$

Teléfono: $(+571) 5878797$, ext. 2991

editorial@usantotomas.edu.co

http://ediciones.usta.edu.co

Corrección de estilo: Yecid Muñoz Santamaría

Diagramación: Alexandra Romero Cortina

Diseño de carátula: Juliana Pardo Torres

Impresión: DGP EDITORES S.A.S.

Hecho el depósito que establece la ley

ISBN: 978-958-782-262-5

E-ISBN: 978-958-782-263-2

Primera edición, 2019

Se prohíbe la reproducción total o parcial de esta obra, por cualquier medio, sin la autorización expresa del titular de los derechos.

Impreso en Colombia $\bullet$ Printed in Colombia 


\section{Contenido}

$\begin{array}{ll}\text { AGRADECIMIENTOS } & 15\end{array}$

$\begin{array}{ll}\text { INTRODUCCIÓN } & 17\end{array}$

Aspectos metodológicos I9

Enfoques de la investigación 19

Material y método $\quad 21$

Objetivos del estudio $\quad 30$

$\begin{array}{ll}\text { Procesamiento de los datos } & 30\end{array}$

PRÁCTICAS FISICODEPORTIVAS: ACTITUD E INTERÉS

DE ESCOLARES COLOMBIANOS 33

Introducción 33

Las habilidades fisicodeportivas y su influencia en la adquisición de hábitos deportivos 34

La recreación en la clase de Educación Física $\quad 47$

NiVEL DE PRÁCTICA FISICODEPORTIVA DE ESCOLARES COLOMBIANOS 75

Las nociones de conducta, estilo y hábito de vida $\quad 75$

Estudios de hábitos y prácticas deportivas $\quad 77$

Formación de hábitos de práctica fisicodeportiva $\quad 79$

Estimación de suficiencia de la práctica fisicodeportiva $\quad 81$

Práctica fisicodeportiva personal que realiza en la actualidad $\quad 82$

Nivel de práctica fisicodeportiva $\quad 83$

Inactividad, sedentarismo y condición física $\quad 85$

Opinión de los jóvenes sobre si hacen suficiente $\begin{array}{ll}\text { o insuficiente práctica fisicodeportiva } & 87\end{array}$ 
Posesión de artículos o materiales deportivos

Valor del deporte para los escolares

Alfabetización Física

Actividad física y ejercicio físico

Impacto del ejercicio físico en la salud

Actividad física, ejercicio físico y su relación con la salud

Lugar en donde se adquieren los hábitos y reconocimiento de los escolares sobre los beneficios de las prácticas fisicodeportivas

Conclusiones

REFERENCIAS

Sobre LOS AUTORES 


\section{Índice de figuras}

Figura 1. Dimensión 1: análisis del perfil demográfico y educativo

Figura 2. Dimensión 2: actitud hacia la práctica deportiva de los estudiantes colombianos de $9 .^{\circ}$ a $11 .^{\circ}$

Figura 3. Dimensión 3: nivel de práctica fisicodeportiva de los escolares

Figura 4. Dimensión 4: recursos físicos y de infraestructura

Figura 5. Alfabetización física

Figura 6. Representación porcentual sexo vs. agrado (ítem 1)

Figura 7. Actividades físicas en la clase de Educación Física

Figura 8. Deportes que practica y que se deben incluir en la clase de Educación Física

Figura 9. Frecuencia de práctica deportiva fuera de la clase de Educación Física

Figura 10. Características de las actividades recreativas

y la recreación 
Figura 12. Actividades recreativas desarrolladas en la clase de Educación Física

Figura 13. Manifestaciones recreativas a incluir en la clase de Educación Física

Figura 14. Motivos por los que se dan las prácticas fisicodeportivas

Figura 15. Preferencia de programas deportivos

Figura 16. El deporte como promotor de la participación cívica

Figura 17. Deportes más relevantes dentro del estudio

Figura 18. Las prácticas deportivas más representativas por zona

Figura 19. Práctica por género de cada disciplina deportiva

Figura 20. Agrado por el ejercicio físico, los juegos y otros deportes entre los practicantes de cada disciplina

Figura 21. Actitudes desarrolladas por los practicantes de fútbol

Figura 22. Actitudes desarrolladas por los practicantes de voleibol

Figura 23. Actitudes desarrolladas por los practicantes de atletismo 66

Figura 24. Actitudes desarrolladas por los practicantes de natación 66

Figura 25. Práctica futura: disposición por deporte 68 
Figura 26. Causas más relevantes que impedirían continuar las prácticas por deporte

Figura 27. Tendencia favorable a utilizar cada disciplina como deporte para todos

Figura 28. Opinión de los escolares practicantes, por disciplina, sobre dónde se adquieren los hábitos para la práctica deportiva

Figura 29. Tendencias de actividades a incluir en la clase de Educación Física, según los practicantes de los principales deportes

Figura 30. Tendencias de corrientes recreativas que quisieran incluir en las clases de Educación Física los practicantes de cada disciplina deportiva

Figura 31. Compañía con que suelen practicar la disciplina deportiva

Figura 32. Interés de participar en actividades fisicodeportivas

Figura 33. Factores necesarios para alcanzar un hábito

Figura 34. Lugar donde se adquieren los hábitos fisicodeportivos

Figura 35. Percepción de la práctica fisicodeportiva de los jóvenes

Figura 36. Práctica fisicodeportiva

Figura 37. Práctica deportiva más frecuente

Figura 38. Representación porcentual sexo vs. estimación de suficiencia (ítem 6) 
Figura 39. Posesión de artículos deportivos

Figura 40. Factores cuantitativos de la actividad física

Figura 41. Factores cualitativos de la actividad física

Figura 42. Características del ejercicio físico según

Torres Guerrero (1996)

Figura 43. Potencialidades del ejercicio físico

Figura 44. Incidencias positivas del ejercicio físico en la salud

Figura 45. Creencia del lugar donde se adquiere el hábito

de la actividad física sistemática

Figura 46. Conocimientos sobre actividad física en los escolares 


\section{Índice de tablas}

Tabla 1. Causas de la insuficiente práctica de las actividades fisicodeportivas

Tabla 2. Valoración del deporte 



\section{Agradecimientos}

Los autores agradecen especialmente a la Universidad Santo Tomás y a Promedios CMAC por el apoyo brindado mediante el ofrecimiento de los recursos y las condiciones requeridas para el buen desarrollo de la investigación. Igualmente, este trabajo no habría sido posible sin la ayuda de todas las personas que se involucraron de forma comprometida, entre las que se encuentran: Juan Carlos Ibarra, Gustavo Adolfo Holguín, Fabio Felipe Buitrago Villafradez, Anna María Moncada, Oscar Miguel Villareal Roche, Paola Andrea Jiménez, Gabriel Antonio Gallego, Emilio Verdugo Camacho, Jeison Salcedo, Lanson May, Oscar Guillermo Goez de los Ríos, Mónica Yhanet Martínez, Katerin Zambrano, José Alfonso, Darío Mendoza Romero, David Gómez, Gina Paola Bonilla Cetina y Jairo Rincón. 



\section{Introducción}

umerosos estudios han determinado que las principales causas
de la aparición de las ECNT (enfermedades crónicas no transmisibles) son la disminución de una vida activa, que incluye la ausencia cada vez más acentuada de la práctica de actividad física en la cotidianidad del ser humano, y la incorporación de una alimentación caracterizada por un excesivo contenido calórico, que potencia factores de riesgo como el sedentarismo, el sobrepeso y la obesidad.

De igual manera, se ha evidenciado el incremento de las cifras de morbimortalidad y el descenso progresivo en la práctica de la actividad física con la edad, que alcanza picos alarmantes en la adolescencia y la edad adulta.

Son varias las investigaciones que coinciden en la necesidad de promover la actividad física desde edades tempranas con la finalidad de prevenir el incremento de las ECNT. En este sentido, la OMS determinó como meta mundial para 2025 una reducción relativa del $10 \%$ en la prevalencia de actividad física insuficiente.

Bajo esta perspectiva, consideramos necesario promover estudios encaminados a identificar las actitudes y tendencias hacia las prácticas fisicodeportivas. En primer lugar, para diagnosticar objetivamente la situación real del interés y el nivel de práctica de los jóvenes como base fundamental de la promoción de hábitos y estilos de vida saludables. En segundo lugar, como insumo fundamental para la definición de las 
políticas deportivas, educativas y sanitarias del país, en el marco de la prevención de la enfermedad a partir de la promoción de las actividades fisicodeportivas como un eficiente activo de salud.

A continuación se presentan los resultados de un estudio de alcance nacional realizado en 29 de los 32 departamentos de Colombia, con una muestra de 2102 escolares de los grados $9 .^{\circ}$ a $11 .^{\circ}$, de edades comprendidas entre los 13 y los 17 años. Se adelantó con el respaldo de la Universidad Santo Tomás de Colombia y Promedios CMAC entre 2014 y 2017.

La investigación fue de tipo descriptivo, para lo cual se empleó como instrumento el Cuestionario de hábitos deportivos de los españoles elaborado por Manuel García Ferrando en el marco del proyecto europeo Compass, en su primera versión de 2000. Este instrumento fue adaptado y validado al contexto colombiano por J. A. Romero y F. Amador en 2007.

Dentro de los aspectos relevantes sobre los cuales se orientó el desarrollo del estudio se encuentran la identificación del interés que tienen los escolares por la clase de Educación Física, los deportes que más practican, las actividades que realizan en el tiempo libre, las personas con las que practican actividades fisicodeportivas, los escenarios más utilizados, los motivos por los cuales seguirían realizando actividades fisicodeportivas, entre otros.

Entre las conclusiones del estudio se encuentra que los jóvenes consideran importante la práctica de las actividades fisicodeportivas porque les permite adquirir una buena forma física y estar con amigos en un ambiente de diversión y entrenamiento. También desearían que se incluyeran en la clase de Educación Física el deporte de aventura, los campamentos y otras actividades físicas. Quienes no realizan ningún tipo de práctica fisicodeportiva manifiestan que es debido a la falta de instalaciones y medios, la ausencia de afición y la escasez de tiempo libre. 


\section{Aspectos metodológicos}

\section{Enfoques de la investigación}

\section{Enfoque epistemológico}

Dara percibir la realidad de las actitudes y tendencias hacia la prác1 tica fisicodeportiva de escolares colombianos de los grados 9. a $11 .^{\circ}$ se propuso el desarrollo de una investigación cuyo fundamento metodológico se centró en identificar los siguientes aspectos: concepciones respecto a las actividades fisicodeportivas, grado de alfabetización respecto a los temas de actividad física, escenarios disponibles para su práctica, entre otros.

Desde esta perspectiva el estudio se realizó con un enfoque epistemológico empírico-inductivo fundamentado en los siguientes criterios:

- Se concibe como producto del conocimiento científico de los patrones de regularidad que explican las interdependencias entre diferentes comportamientos o elementos. En tal sentido, dentro del marco de la investigación la completa y compleja red de información arrojada por los escolares se organizó en patrones de regularidad que luego fueron analizados estadísticamente, lo que permitió desarrollar inferencias probabilísticas sobre las actitudes y tendencias hacia las prácticas fisicodeportivas.

- Los medios para el acceso al conocimiento y los instrumentos definidos para su producción y validación, no pueden ser otros que los sentidos y sus prolongaciones (instrumentos de observación y medición) debido a que los patrones de regularidad 
se captan a través del registro de repeticiones de eventos. Por tanto, es el método inductivo, sustentado en el poder de los instrumentos sensoriales y en el valor de los datos de la experiencia (de allí el término empirista), el sistema de operaciones privilegiado dentro de este enfoque.

El conocimiento, entonces, correspondería en este caso a un acto en el que descubrimos las actitudes y tendencias hacia la práctica de las actividades fisicodeportivas de los escolares a través de sus patrones de comportamiento en la realidad. Por ende, la investigación se centró principalmente en la cuantificación de los contenidos fisicodeportivos más representativos y en la caracterización y el análisis de las tendencias hacia contenidos diferenciados en deportes y actividades recreativas y físicas.

\section{Enfoque metodológico}

Se estructuró para el desarrollo de esta investigación un enfoque cuantitativo que permitió fundamentar el proceso estableciendo primero las formas de medición de variables previamente determinadas por los investigadores. A continuación se procedió a la aplicación de técnicas estadísticas para conocer el comportamiento de dichas variables y sus relaciones, y así identificar las actitudes y tendencias de los escolares hacia las prácticas fisicodeportivas.

\section{Tipo de investigación}

El estudio realizado es de tipo descriptivo, en cuanto que los investigadores interactuaron con la muestra a partir de la aplicación de una encuesta y la información suministrada fue objeto de análisis sin modificarla. Esto permitió identificar las actitudes y tendencias hacia la práctica de las actividades fisicodeportivas por parte de los escolares colombianos de los grados $9 .^{\circ}$ a $11 .^{\circ}$. 


\section{Diseño de la investigación}

El diseño fue de tipo no experimental transeccional correlacional. Eso permitió describir las relaciones entre dos o más variables en un momento determinado. Los diseños transeccionales descriptivos tienen como objeto indagar la incidencia y los valores en los que se manifiestan una o más variables (Hernández Sampieri et al. 2001).

\section{Material y método}

\section{Sujetos: población y muestra}

El universo de estudio comprende la población colombiana entre 13 y 17 años de edad que, de acuerdo a lo proyectado por el DANE (Departamento Administrativo Nacional de Estadística), para 2017 correspondía a un total de 4278852 jóvenes, de los cuales $48.94 \%$ eran mujeres.

La población de referencia correspondió a todos los niños y jóvenes entre 13 y 17 años pertenecientes a los grados 9. ${ }^{\circ}$ a $11 .^{\circ}$ del sistema educativo colombiano ubicados en los cascos urbanos.

Considerada esta población se aplicó la siguiente fórmula para la estimación de un tamaño muestral:

$$
n=\frac{z_{\alpha / 2}^{2}{ }^{*} p q}{F E^{2}}
$$

Donde:

$\mathrm{n}=$ tamaño de muestra.

$\mathrm{z}$ = valor de la normal para un error alfa a dos colas que corresponde a 1.96

$\mathrm{p}=$ proporción esperada.

$\mathrm{q}$ = inverso de la proporción.

$\mathrm{FE}=$ factor de error. 
Un estudio previo llevado a cabo en cinco ciudades de Colombia reportó una prevalencia de actividad física de un 19.8 \% como máximo en 2010 (Piñeros y Pardo, 2010). A partir de este dato se estima un tamaño muestral con un factor de error de $2 \%$, lo que corresponde a un tamaño muestral mínimo de 1525 jóvenes y un ajuste por pérdidas del $30 \%$.

Dado este tamaño muestral el proceso de selección de muestras se dividió en tres etapas, cada una de las cuales determinó una unidad de muestreo como se presenta a continuación:

- En la primera etapa se seleccionaron 29 de los 32 departamentos (conglomerados) de cada una de las regiones del país. En el proceso de selección el factor determinante fue la facilidad de acceso para la aplicación del instrumento.

- En la segunda etapa se seleccionaron de forma aleatoria simple 74 municipios (unidades primarias de muestreo) y dentro de ellos, 361 instituciones educativas en total.

- En la tercera etapa se seleccionaron los individuos según estratos, de forma aleatoria simple, de acuerdo al número de jóvenes matriculados en cada uno de los centros educativos.

El total de la muestra fue de 2102 sujetos del grupo etario de los 13 hasta los 17 , todos estudiantes colombianos de $9 .^{\circ}, 10 .^{\circ}$ y $11^{\circ}$, de los cuales 1075 pertenecían al sexo masculino y 994 al femenino.

\section{Material: instrumento utilizado para la recolección de información}

Para la recolección de la información se empleó como instrumento el cuestionario de hábitos deportivos elaborado por Manuel García Ferrando, validado y adaptado al contexto colombiano por Romero y Amador (2007). En la fase de validación el cuestionario fue aplicado manteniendo la redacción, la composición y el orden original. 
Es importante señalar que el estudio realizado por García Ferrando tiene una aplicación cada cinco años desde 2000, así que se reconoce como una de las investigaciones más consistentes en el tiempo. El cuestionario consta de 64 preguntas agrupadas en veinte variables que se integran en las siguientes dimensiones:

- Tiempo libre, actividades de ocio y deporte.

- La práctica deportiva de la población española.

- La dimensión subjetiva de la práctica deportiva.

- La dimensión de las prácticas deportivas de la competición y la aventura en la naturaleza al paseo y al deporte para todos.

- La imagen del deporte español.

- Las dimensiones asociativas de la práctica deportiva y el deporte como consumo y espectáculo de masas.

- Los determinantes de las prácticas deportivas en la posmodernidad.

\section{Validación del cuestionario}

El cuestionario fue validado y adaptado por Romero y Amador (2007) en su composición, orden y redacción, considerando elementos terminológicos y lingüísticos propios del contexto colombiano.

El cuestionario de aplicación en el presente estudio está conformado y organizado por 39 ítems que abordan los siguientes cinco ejes centrales denominados dimensiones:

- Dimensión 1: perfil demográfico y educativo. Constituida por cinco preguntas que indagan sobre el género del sujeto, su edad, 
el grado académico que cursa, el departamento y el municipio donde reside. Incluyó un espacio para indicar un número de contacto a través del cual, posteriormente, se realizó la validación de la información.

Esta dimensión está ubicada al final del cuestionario y corresponde a las preguntas que van de la 34 a la 38 y la pregunta 39 no tipificada.

Figura 1. Dimensión: análisis del perfil demográfico y educativo

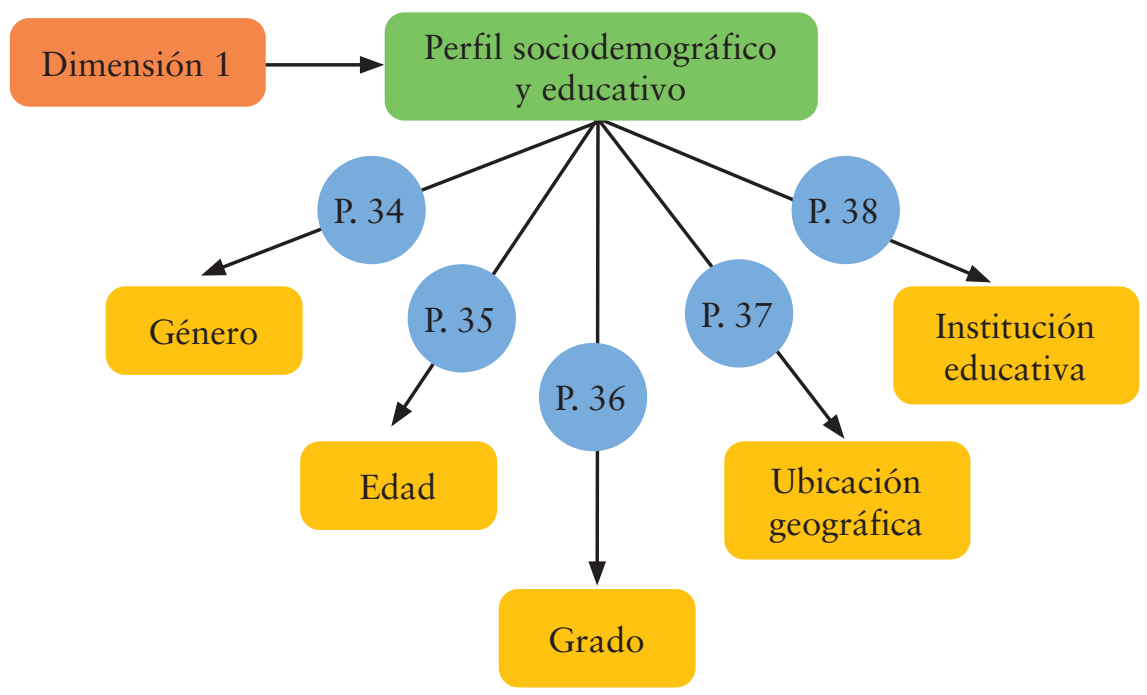

- Dimensión 2: actitud hacia la práctica fisicodeportiva. Relaciona todas las preguntas sobre la actitud hacia la práctica fisicodeportiva. Se concentró en identificar las siguientes subcategorías:

- Comprensión de los estudiantes de una educación física de calidad.

- Interés por las prácticas fisicodeportivas.

- Valoración de las prácticas fisicodeportivas.

- Creencias sobre las prácticas fisicodeportivas. 
Aspectos METOdológicos

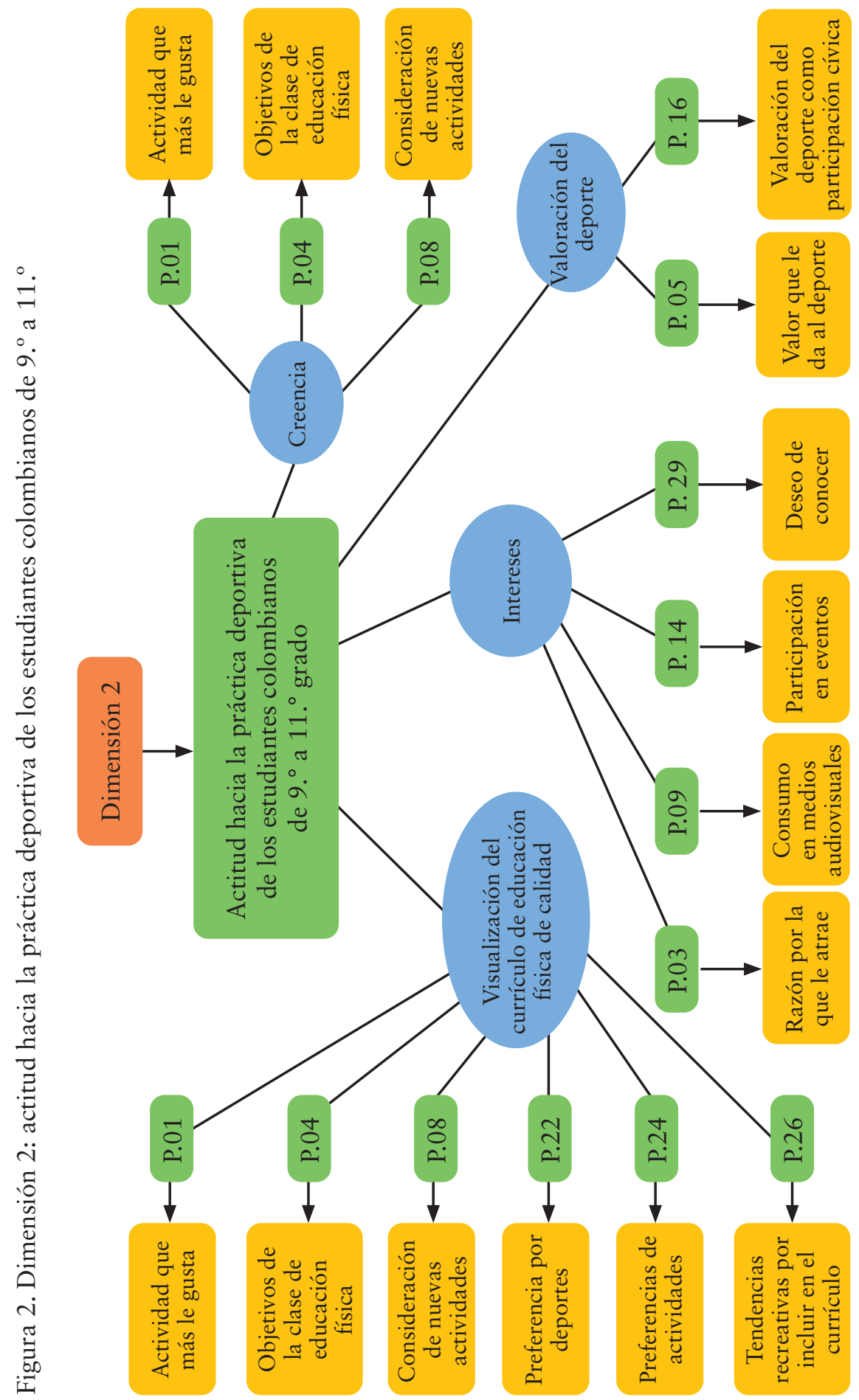


- Dimensión 3: nivel de práctica fisicodeportiva de los escolares. Buscó identificar el nivel de práctica fisicodeportiva de los escolares, las prácticas pasadas y, para el caso de quienes manifestaron no practicar actividad física alguna, las razones de esa decisión. En este sentido, se presentaron las siguientes cuatro subcategorías:

- Práctica fisicodeportiva actual.

- Frecuencia de práctica fisicodeportiva.

- Acceso a implementos deportivos en el hogar.

- Prácticas fisicodeportivas realizadas en el pasado y motivos de abandono.

- Dimensión 4: infraestructura para la práctica fisicodeportiva en horas extracurriculares. Orientada a identificar los recursos de infraestructura en donde realizan la práctica fisicodeportiva los escolares en horas extracurriculares, así como la opinión que tienen de dichos espacios físicos y a quién creen que corresponde la construcción y mantenimiento de los proyectos de infraestructura deportiva. Este aspecto se analizó a partir de las siguientes tres subcategorías:

- Instalaciones utilizadas actualmente para las prácticas fisicodeportivas.

- Accesibilidad a la infraestructura deportiva.

- Proyección de nuevas instalaciones para la práctica fisicodeportiva. 


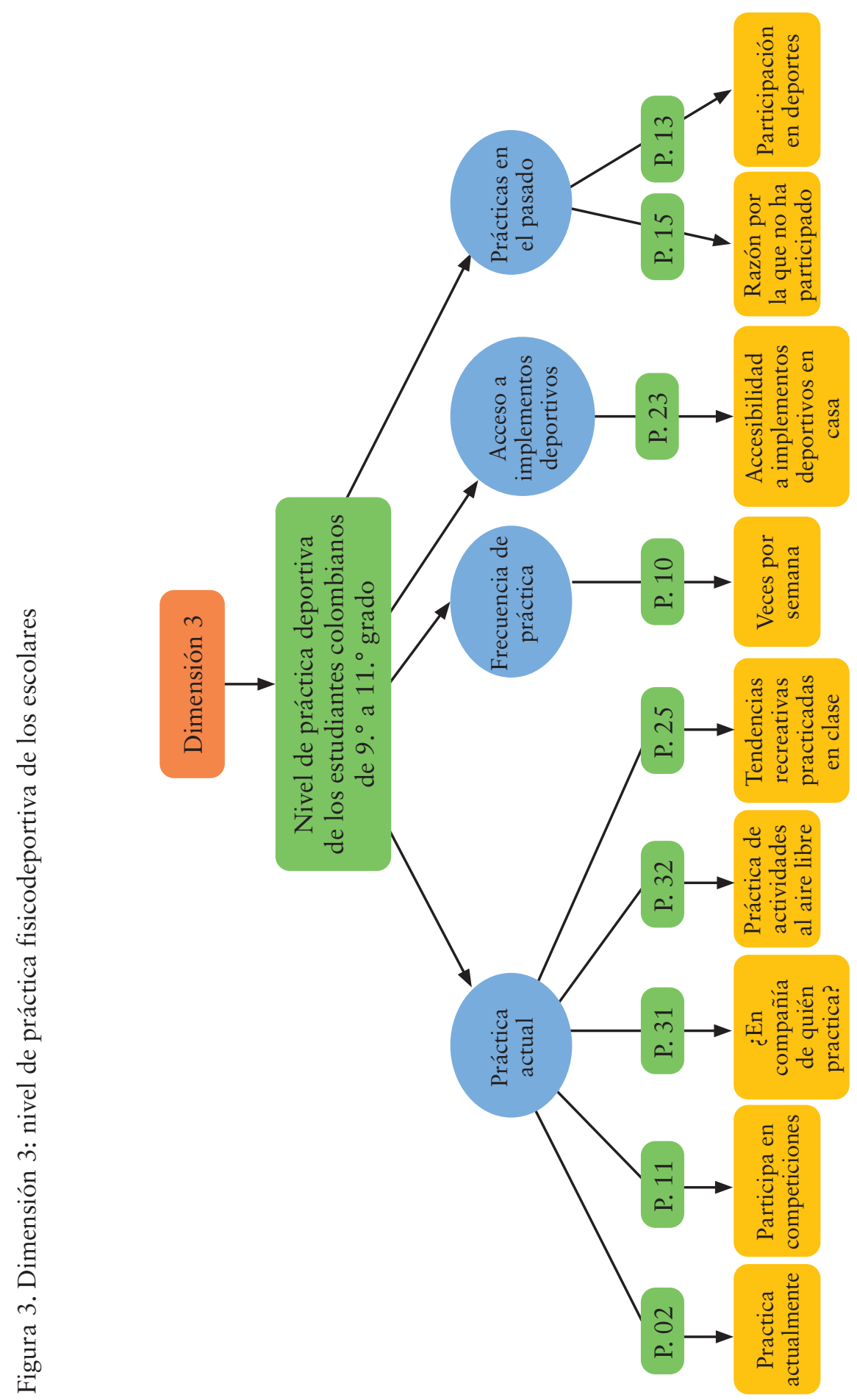


Figura 4. Dimensión 4: recursos físicos y de infraestructura

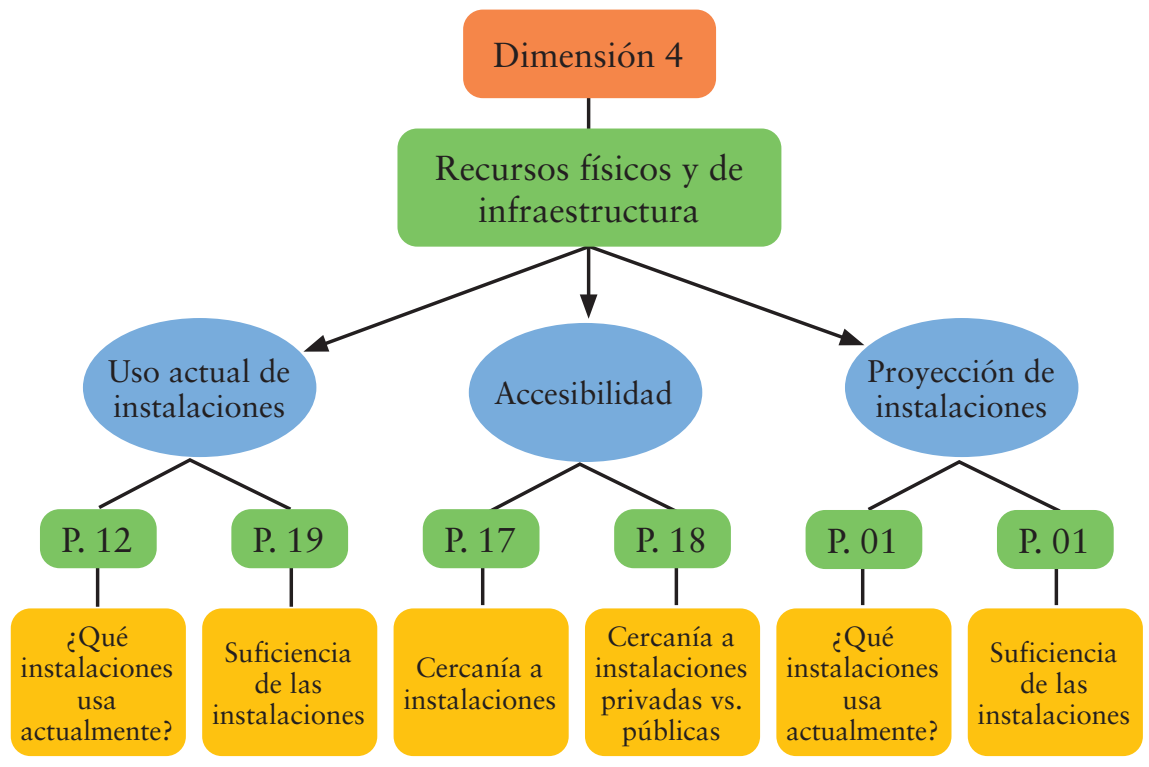

- Dimensión 5: alfabetización física de los escolares. Se centra en identificar si el escolar ha recibido educación sobre la actividad física y si reconoce los beneficios de esta y su incidencia como práctica regular durante toda la vida.

Figura 5. Alfabetización física

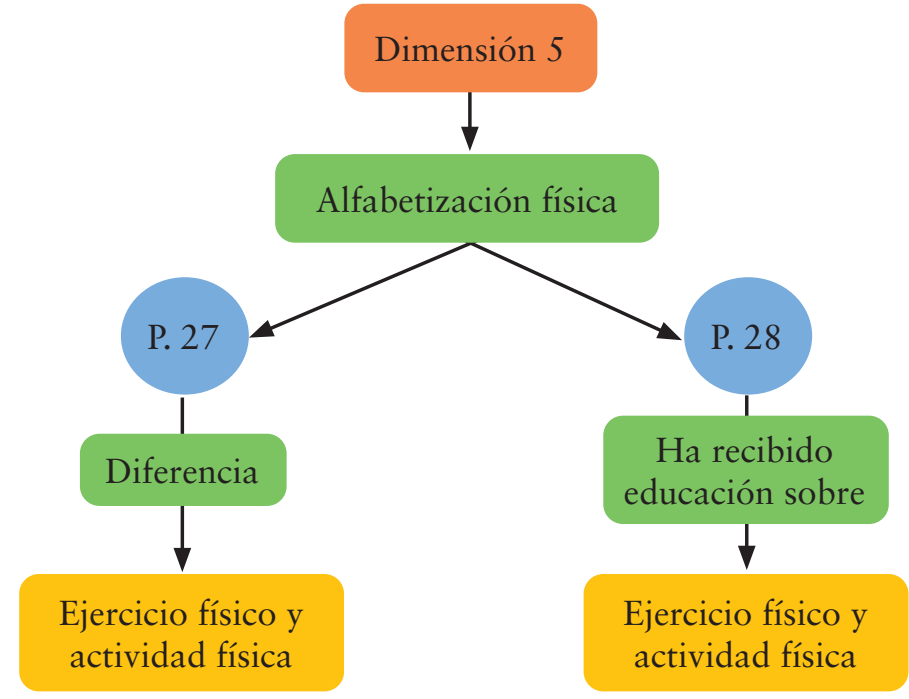




\section{Lugar en donde se realizó la investigación}

La investigación se realizó en Colombia en los departamentos de: Amazonas, Antioquia, Arauca, Atlántico, Bolívar, Boyacá, Caldas, Caquetá, Casanare, Cauca, Cesar, Córdoba, Cundinamarca, Chocó, Guajira, Guaviare, Magdalena, Meta, Nariño, Norte de Santander, Putumayo, Quindío, Risaralda, San Andrés Islas, Santander, Sucre, Tolima, Valle del Cauca y Bogotá.

En cuanto a la infraestructura usada para preparar la recolección de la información, su validación estadística y el almacenamiento de los archivos en físico, se adecuó un espacio en las instalaciones de Promedios CMAC y otro en la Universidad Santo Tomás, sitio de reunión del grupo Gicaeds y Cuerpo, Sujeto y Educación en la ciudad de Bogotá, Colombia.

\section{Tiempo destinado a la investigación}

El tiempo destinado al desarrollo de la investigación incluyó la preparación, la aplicación, la organización, la depuración, el análisis, la elaboración del informe final y la divulgación de los resultados.

La recolección de los datos estuvo supeditada a las diferentes fases del proceso de investigación: selección de la muestra, determinación de las unidades de muestreo (municipios y centros educativos), preparación para la aplicación del instrumento y coordinación de esta, recolección y procesamiento de la información, análisis y discusión de los resultados y elaboración del informe final. El proceso inició el segundo semestre de 2014 y finalizó el primer semestre de 2017. 


\section{Objetivos del estudio}

\section{Objetivo general}

Identificar las actitudes y tendencias hacia la práctica fisicodeportiva de los escolares colombianos entre $9 .^{\circ}$ y $11 .^{\circ}$.

\section{Objetivos específicos}

- Describir el perfil sociodemográfico y educativo de los escolares colombianos entre $9 .^{\circ}$ y $11 .^{\circ}$.

- Identificar las actitudes hacia la práctica deportiva de los escolares colombianos entre $9 .^{\circ}$ y $11 .^{\circ}$.

- Describir el nivel de práctica deportiva de los escolares colombianos entre $9 .^{\circ}$ y $11 .^{\circ}$.

- Identificar las tendencias hacia la práctica fisicodeportiva de los escolares colombianos entre $9 .^{\circ}$ y $11 .^{\circ}$.

\section{Procesamiento de los datos}

Las actividades propias del procesamiento de datos se iniciaron con el entrenamiento del personal encargado de la codificación y digitalización. Se organizaron y enumeraron los cuestionarios en el centro de registro y digitalización a medida que fueron llegando. Se elaboraron cuatro tablas para descargar la información de los cuestionarios. En dichas tablas se registraron las respuestas abiertas (siete en total), las cuales fueron categorizadas y posteriormente ordenadas de acuerdo a la frecuencia de la respuesta dada. Las tablas elaboradas fueron las siguientes: 
- Tabla de categorización de respuestas. Se utilizó para descargar todas las respuestas abiertas de cada una de las muestras, de tal manera que se pudieran identificar las diferentes categorías.

- Tabla de registro de respuestas para las preguntas abiertas en las que se identificaron las respuestas de cada uno de los entrevistados.

- Tabla de registro de frecuencia de respuestas de las preguntas abiertas, usada para establecer una categorización de las respuestas en orden de mayor a menor frecuencia.

- Tabla para la identificación numérica de las categorías de respuestas a registrar, mediante la cual se asignó un número a cada respuesta para establecer una categorización numérica que facilitara su respectivo registro.

Se digitalizó la información en una plantilla elaborada bajo el formato Access y preparada para los registros en una matriz, que posteriormente se trasladó al programa estadístico spss versión 20 .

\section{Resultados, análisis y discusión del estudio}

Los resultados obtenidos se presentan por cada una de las cinco dimensiones; las acompañamos con gráficas y en algunas de las preguntas con tablas.

En aras de una mayor claridad expositiva, junto a los resultados se adjuntó la correspondiente discusión teniendo como referencia general comparada el estudio homólogo de Romero y Amador (2007), además de las siguientes investigaciones: Bizkaia (2002); Dishman et al. (2005); Escudero López y Serra (1992); García Ferrando (2000); Guillén, Weis y Navarro (2005); Haverly y Davison (2005); Ispizua (2003); Malina, Bouchard y Bar-Or (1991); Martínez Baena et al. (2012a; 2012b); Martínez Gómez et al. (2009); Otero (2004); Piéron (2002); 
Ponseti (1998); Ruiz y García (2004); Utter et al. (2006); Vera et al. (2010).

\section{Tratamiento estadístico}

El tratamiento del proceso estadístico del presente estudio se realizó sobre la base de la propuesta de Romero y Amador (2007). El propósito fue establecer los elementos necesarios para contrastar los resultados de los dos estudios y consolidar una caracterización de las tendencias y actitudes hacia las prácticas fisicodeportivas de los escolares y universitarios colombianos en edades comprendidas entre los trece y los veinticinco años. Desde esta perspectiva, el proceso estadístico implicó tres momentos: recolección de la información, tabulación y procesamiento de esta e interpretación y análisis. El momento destinado a la recolección y tabulación de la información utilizó en primer lugar el cuestionario y un formulario de captura del instrumento diseñado en Access 2010.

El instrumento Access permitió construir una base de datos que posteriormente migró a la aplicación spss versión 20. Con esta información se generaron los insumos para realizar el análisis e interpretación de los resultados obtenidos. 